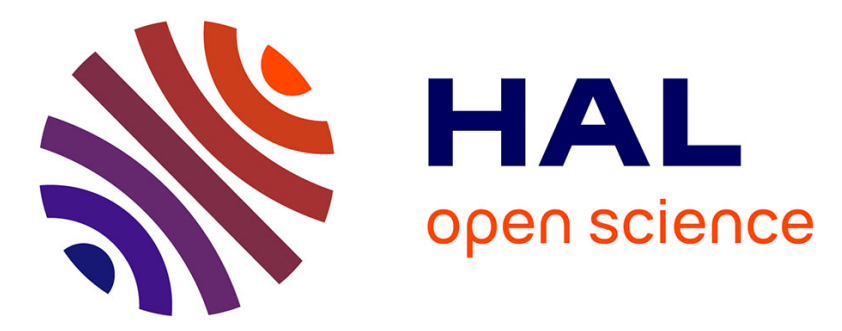

\title{
A conceptual model for SMEs mechatronics supply chain.
}

\author{
Jihène Tounsi, Julien Boissiere, Georges Habchi
}

\section{To cite this version:}

Jihène Tounsi, Julien Boissiere, Georges Habchi. A conceptual model for SMEs mechatronics supply chain.. International Journal of Computer Aided Engineering and Technology, 2010, 2 (4), pp. 371387. hal-00403793

\section{HAL Id: hal-00403793 \\ https://hal.univ-smb.fr/hal-00403793}

Submitted on 9 Jan 2013

HAL is a multi-disciplinary open access archive for the deposit and dissemination of scientific research documents, whether they are published or not. The documents may come from teaching and research institutions in France or abroad, or from public or private research centers.
L'archive ouverte pluridisciplinaire HAL, est destinée au dépôt et à la diffusion de documents scientifiques de niveau recherche, publiés ou non, émanant des établissements d'enseignement et de recherche français ou étrangers, des laboratoires publics ou privés. 


\title{
J. Tounsi
}

University of Savoie, SYMME-Polytech'Savoie

12, chemin de Bellevue BP 80439

74940, Annecy-Le-Vieux

E-mail: jihene.tounsi@univ-savoie.fr

\section{J. Boissière}

University of Savoie, LISTIC-Polytech'Savoie

12, chemin de Bellevue BP 80439

74940, Annecy-Le-Vieux

E-mail: julien.boissiere@univ-savoie.fr

\section{G. Habchi}

University of Savoie, SYMME-Polytech'Savoie

12, chemin de Bellevue BP 80439

74940, Annecy-Le-Vieux

E-mail: georges.habchi@univ-savoie.fr

\begin{abstract}
Nowadays, in order to meet the demands of the global dynamic market and eliminate its disturbances, the supply chain and its integration across different organizations has become a competitive business advantage along the production processes. In particular, the mechatronic industry is very sensitive to these requirements. Our research deals with modelling the supply chain in order to manage and implement a simulation platform. Basically, we develop a modelling solution for an industrial environment composed of mechatronic Small and Medium Enterprises located in Savoie (France). The study is based on elements from real applications and the SMEs network organization. An analysis of the domain helped us to develop concepts and a modelling approach adapted to these companies. In this work, we present the area studied, the methodology used to construct the conceptual model of the supply chain, its structure and management rules. Then, we present the model obtained. Finally, we apply this conceptual model to a case study.
\end{abstract}

Keywords: SMEs, supply chain, modelling methodology, conceptual model, simulation

Reference to this paper should be made as follows: Tounsi, J., Boissière, J. and Habchi, G. (2009) 'A conceptual model for SMEs mechatronics supply chain', Int. J. Computer Aided Engineering and Technology, Vol. x, No. x, pp. X-Xx.

Bibliographical notes: Jihène Tounsi is a PhD student in the SYMME Laboratory, University of Savoie, France. She is pursuing her PhD studies in Industrial Engineering. She has submitted 5 papers to international conferences including one in the French language.

Julien Boissière is an Assistant Professor at the Department of Quality, Logistics and Industrial Organisation (QLIO). His research interests are mainly optimisation and simulation, performance evaluation and multi-criteria decision making in supply chain contexts. He has published a dozen of international research papers for journal or conferences.

Georges Habchi is a Full Professor at the Department of Quality, Logistics and Industrial Organisation (QLIO), and is the Director of the Doctoral School of Sciences and Engineering of Systems, Environment and Organisations, University of Savoie. His research interests mainly deal with control, modelling and simulation of supply chain and production systems. He has published more than 80 research papers in international journals and conference proceedings. 


\section{Introduction}

The integration of the supply chain between the various actors is a major strategic challenge and a strong competitive advantage. Indeed, the supply chain is a complex macro-system, first due to the variety of the organizational structures involved and the relationships between them, and secondly due to the strategic decisions that must be made.

Small and Medium Enterprises (SMEs) evolve in an unstable and complex economic environment. In order to survive and to be able to support the supply chain's requirements (internal factors: influent actors, delay, consumer satisfaction, etc.) and market requirements (external factors: competition, unpredictable mutation, etc.), SMEs have to collaborate together to achieve their goals without losing their autonomy and identity (Villarreal Lizarraga et al., 2005; Julien, 1997).

The industrial environment of Savoie, corresponding to our research field, consists of numerous productive SMEs in the mechatronic industry working together to achieve a common goal in a complex global supply chain. We are interested in mechatronic SMEs as represent a relatively new concept that has emerged in several industrial areas that need to integrate mechanical, electronics and computer knowledge simultaneously into production. These SMEs are an economic and a competitive advantage for the survival and the prosperity of the region's economy.

Due to the complexity and heterogeneity of a supply chain composed of SMEs and the lack of work in this area, we have focused our research in this direction. For all these reasons, we propose to study the structure and the SMEs dynamic behaviour in their overall supply chain.

In this paper, we present an analysis of the industrial environment as well as the modelling approach in the context of mechatronic SMEs. Thus, this work is divided into three main parts. The first part presents a study of the existing situation concerning theories discussed in literature. In the second part, we define the modelling methodology which is inspired from research in literature. Then in a third part, we develop a conceptual model representing our vision of supply chain. The last section concludes the paper by developing the model based on a real industrial case.

\section{Literature overview}

In this section, we define the place of SMEs in the supply chain and in supply chain management (SCM) based on the literature. We also present the problem which encouraged us to try to reduce the complexity of supply chain in this context. Moreover, in the last part we present the different modelling visions in the supply chain and SCM field.

\subsection{Supply chain and SCM}

The supply chain concept was born in the 90s when the management techniques in the business world had evolved from separate logistics to collaborated logistics. According to Christopher (Christopher, 1992), the supply chain can be seen as a network of companies involved in the different processes and activities both upstream and downstream that create value in the form of goods and services brought to the final consumer.

As such, a company's success lies in its ability to integrate the managerial processes of the supply chain but also to coordinate with other actors (Drucker, 1998; Lambert and Cooper, 2000).

According to Bagchi and Skjoett-Larsen (Bagchi and Skjoett-Larsen, 2005), the integration can be defined as a complete collaboration between decision-making levels: strategic, tactical and operational level. It is based on 5 interrelated key points:

- Sharing of information and communication

- Collaboration among the actors of the supply chain

- Collaboration leading to sharing risks, costs and benefits

- Sharing of technical expertise, ideas and institutional cultures

- Organization 
In fact, to be able to talk about a successful SCM tool, we must consider the supply chain structure, the processes that add value and the management components that define how to manage the entire supply chain (Cooper et al., 1997).

\subsection{SMEs role in the supply chain}

On the one hand, we have found a lot of work in literature dealing with the integration of the supply chain from a big company's point of view. In this case, the company is considered as the heart of the supply chain structure. This point of view mainly focuses on the producing company's performance and pushes other companies to fulfil the requirements of this major company or to synchronize with its manufacturing system.

On the other hand, SMEs are embedded in a complex and unpredictable system subject, in the same time, to economic and social mutations. According to Julien (Julien, 1997), to find an equilibrium between big companies and SMEs, the SMEs must maintain and even improve their competitiveness in an increasingly competitive market. This competitiveness can be achieved by the control of information and processes but also thanks to a network structure.

Indeed, a SME in a supply chain becomes more efficient when it evolves into a network. Mallidi et al (Mallidi et al., 1999) explain the advantages for SMEs to cluster into a SMEs network as it provides:

- Access to new markets by manufacturing new products that are out of reach of a single SME

- Increased productivity by grouping and aligning the productive capacity of each actor of the network

- Improved responsiveness thanks to common responses to the disturbances which are unbearable by a single actor

- Improved use of resources by avoiding functions duplication in the cluster.

The theory of a SMEs network, which is based on collaboration, cooperation and coordination, is a crucial roundabout for its management strategy. These alliances will reduce their vulnerability which stresses SCM integration in an overall supply chain context. This philosophy is explained by Assens (Assens, 1994): "the enterprises network is based on an autonomous and independent SMEs network which interacts in the context of mutual trust relationships to reduce the uncertainty of their environment. During these local interactions, a global organization form emerges in the absence of a central regulatory entity."

\subsection{Modelling approach}

Modelling is a mechanism that reflects the real system which, when coupled with simulation, provides a very powerful decision-making tool. This tool can consider the dynamics of the system and the behaviour of different autonomous actors.

The literature is unanimous on the positive role of modelling and simulation (M\&S) in the study, analysis and performance evaluation of complex systems. For example, some authors (Lee et al., 2002; Longo and Mirabelli, 2008; Ingalls, 1998) highlight the features and advantages of a decision-making tool based on the modelling and discrete event simulation. The M\&S approach has been adopted in several works in order to reduce the complexity of the SCM and evaluate its performance (Bagchi et al., 1998; Labarthe et al., 2007).

Indeed, the simulation translates the supply chain conceptual model and recreates the complexity and highly stochastic environment of a real supply chain. The conceptual model defines the concepts (entities involved) and parameters that give a supply chain manager the possibility to analyze different scenarios by changing the input parameters (Longo and Mirabelli, 2008).

Several orientations of supply chain modelling arise when studying literature. On the one hand, Thierry (Thierry, 2003) provides two orthogonal visions: the Product Vision and the Company Vision.

- The Product Vision considers the supply chain dedicated to the product (or product line); from the raw materials to the final customer. It focuses on the product flow to define the starting and end points of the supply chain. Rota et al (Rota et al., 2002) define the supply chain dedicated to a 
product (or family of products) as "all companies involved in the processes of manufacturing, distribution and sales of the product, from the first of suppliers to the ultimate customer".

- The Company Vision is centred on the company rather than on the product. In this case, the object is the supply chain of the company. This vision focuses on the company modelling, as an organization within a supply chain with all the products it manufactures and all the relationships it might have with other actors in the supply chain. Many models have been proposed in this perspective among them the famous SCOR model, or the model proposed by Kearney (Kearney, 1994).

These two visions are disjointed. They can not be handled at the same time due to the number and the complexity of the separate supply chains crossovers. On the other hand, two other visions can be found in the literature: the Process Vision and the Structure Vision.

- The Process Vision is based on the process classification according to the level of decision-making (Chopra and Meindl, 2001; Stevens, 1989): strategic, tactical and operational. This point of view deals with identifying processes that operate in the supply chain and their levels of decision-making.

- The Structure Vision has been proposed by Cooper et al. (Cooper et al., 1997). It considers the structure of the supply chain which is made up of:

- Actor's type: in a model, the actors who have an important decision-making role (primary actors) should be identified as well as those who synchronize activities without taking part in the decision-making (secondary actors).

- $\quad$ Network structure: the structure is defined in two dimensions: horizontal and vertical. The horizontal dimension defines the different roles that actors can have in the supply chain (suppliers, producers, etc.). The vertical dimension defines the number of actors for each role.

- $\quad$ Relationship characteristics: the actors share several processes that define their behaviour characteristics in the supply chain (management process, control and monitoring or outsourcing).

These two visions are complementary: the choice of one does not exclude the use of the other one in the same time. The first defines the supply chain from a process point of view and the second from an organizational point of view.

\section{Research scope}

According to a survey that we conducted in the mechatronic companies in Savoie (France) and using previous investigations carried out, we have found out that the industrial environment is mostly composed of subcontracting SMEs specialized in mechatronics. Several features of mechatronic supply chain in the region have been drawn from the questionnaire:

- Complexity: the supply chain is composed of several autonomous and independent actors. The relationships between the actors differ depending on the objectives and geographical location. In fact, there are network companies working together to achieve a given process. Each actor may be seen as an expert actor for a specific activity.

- Decentralization/outsourcing: it is a strategic decision due to globalization and the desire to reduce costs. The SMEs studied are mostly subcontractors, which are not necessarily located in the same region or even in the same country.

- Lack of visibility in the overall supply chain: SMEs collaborate by linking their activities to achieve an objective or a process. Or, as a result of the previous characteristics (decentralization and outsourcing), the supply chain is divided into many sites spread over several geographic locations depending on their purpose or activity in the overall supply chain (supply, manufacture, distribution, etc.). These sites only have a local visibility but are coordinated with other sites through product flows. 
In the following section, we discuss the scope of our research, namely a supply chain in SMEs mechatronics. M\&S of the supply chain behaviour in the SMEs mechatronic context is a challenge and a growing need expressed by professionals.

Our choice of the M\&S approach is justified, on the one hand, by the lack of analytical models to model the dynamic of the overall supply chain with all its complexity and on the other hand, by the power of simulation to manage stochastic behaviours of the supply chain (Lee et al., 2002).

\section{Modelling methodology}

In the previous part, we have defined the different points of view adopted to model a supply chain. In this section, we present the modelling methodology we followed. It combines simultaneously the Structure Vision with the Process Vision. These two visions are based on the Product Vision which represents our modelling framework.

To generate the conceptual model, we propose an incremental methodology based on these three visions (Figure 1). In each step, a vision is applied to construct and to refine the conceptual model. The result of each step (intermediary model) will be the input of the next one. Hence, at the end of the three steps, the architecture of the conceptual model is obtained.

Figure 1 Methodology framework

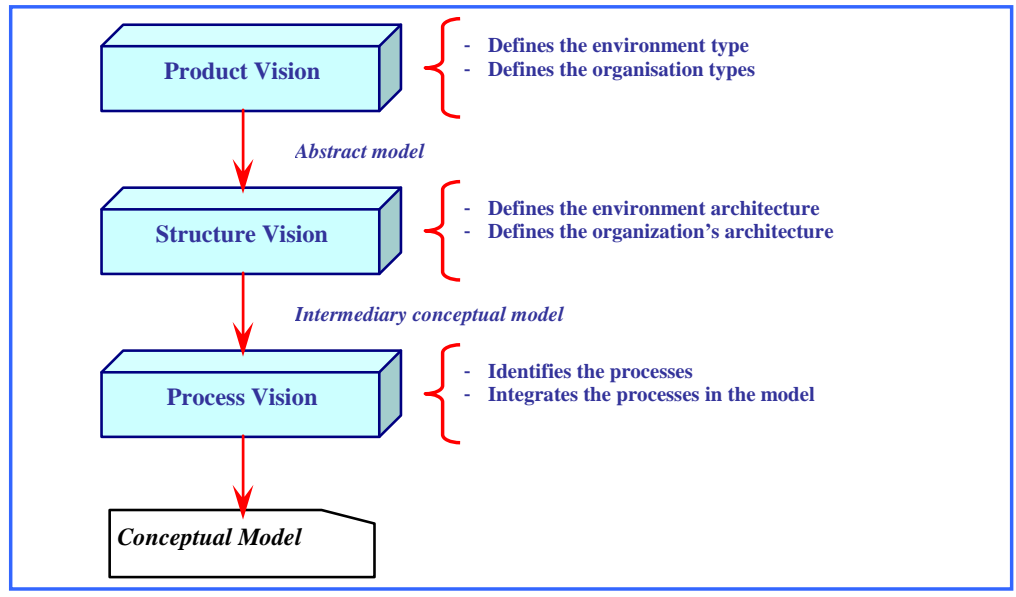

\subsection{Product Vision}

This vision leads us to define a first abstract model of the supply chain. This first model follows the product life cycle. Thus, by adopting a product point of view, we identify the frame of the supply chain which consists of:

- The environment characterized by the flow circulation and the different steps of the product's transformation as well as the related disturbances.

- The organizations needed to guarantee the physical flow management. They carry out one or several transformation stages of the products. Knowing that the application context is a supply chain essentially composed of SMEs, the organizations involved can be a network of firms that collaborate to accomplish one or several transformation stages.

\subsection{Structure Vision}

On the basis of the abstract model provided by the previous step, the Structure Vision details the organizations that are involved and also the physical environment. This vision is based on the identification of three essential elements: involved entities, vertical and horizontal hierarchy as well as the organizational relationships between them. 
- The structure vision of the environment is the part containing the physical flow. Then, we need to model the product flow and the resources needed to achieve its transformation.

- The structure vision of organizations consists in identifying the actors who take part in the network.

- Actors will be prioritized in the organization according to their involvement in the level of decisionmaking and the tasks that will be awarded. The information flow management will be different depending on the level of decision-making.

The application of this step allows us to build a more detailed intermediate model.

\subsection{Process Vision}

While implementing Process Vision, various categories of processes can be identified. This can be done according to the level of decision-making, but also depending on the actors' relationships. These relationships can be classified into two categories:

- Management and control: contains the processes that ensure suitable decision implementation in the perspective of a continuous improvement of processes in terms of added value.

- Synchronization: contains processes for exchanging information and physical flows according to a processes scheme developed and already predefined by the decision-making layers.

At the end of this step, we get a refined conceptual model of the supply chain.

\section{Conceptual model generation}

In this section, we present the generation of the conceptual model as applied to SMEs context following the different phases of the proposed methodology. The objectives are to identify the concepts of the model, its architecture and processes.

\subsection{Applying "Product Vision"}

By applying the Product Vision method, we get a first abstract model of the overall supply chain (Figure 2).

Figure 2 Abstract model of supply chain

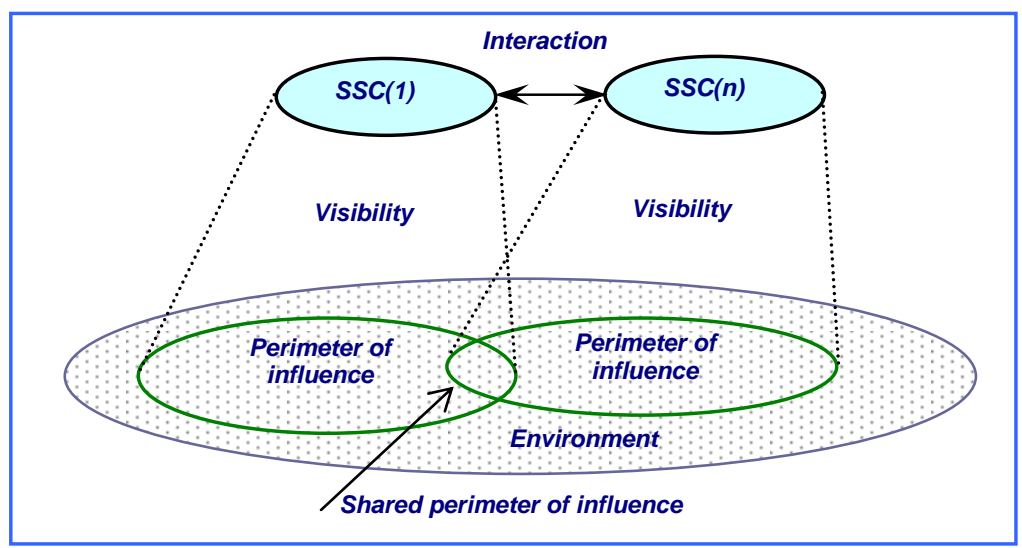

This model consists of:

- An environment defined by the space allocated to the product flow and management between the elements of the supply chain known by the internal resources as well as all the external elements that can influence the chain activity. 
- Sub Supply Chains (SSCs): each SSC represents a group of SMEs which collaborate to achieve an internal aim and/or the overall objective of the overall supply chain. The SSC is responsible for the processing of the product in a certain stage of its life cycle.

- A perimeter of influence (boundary) representing the visible environment for the SSC and on which it can act by internal conferring (if the action does not disturb the environment located outside of the limit of its visibility) or by conferring with other SSCs.

- A shared perimeter of influence representing the area of flow transfer between two SSCs. It is a shared zone where two SSCs coordinate their activities to allow the transfer of flow.

This abstract model is the first result of applying Product Vision. In the following paragraphs, we detail this model by applying the other two visions to structure the architecture and the processes of the abstract model.

\subsection{Applying "Structure Vision"}

In this stage of modelling, we detail the internal architecture of the SSC and the environment which is visible through its perimeter of influence.

In the conceptual model, a SSC defines a network of companies that collaborate to achieve common goals (internal and/or external). It consists of three layers representing the different levels of decisionmaking (Figure 3). Each layer is composed of several concepts and plays a specific role in the SSC.

Figure 3 Layers of the SSC

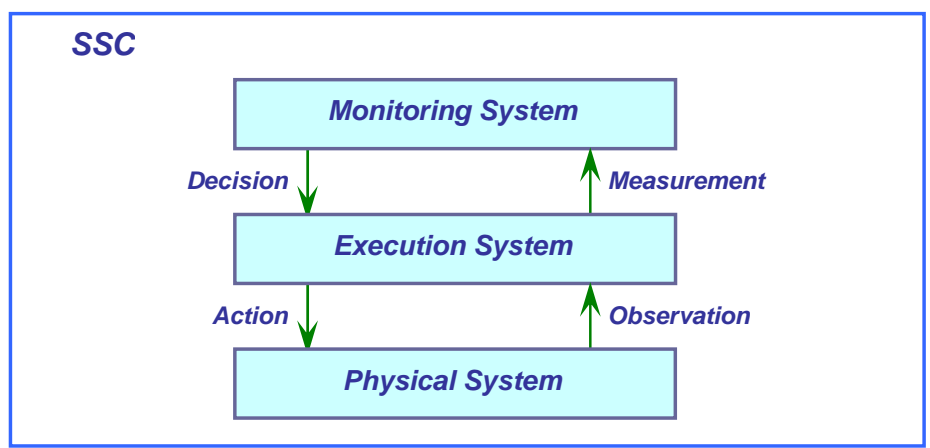

The first top layer called the Monitoring System is the intelligent layer of the SSC. It ensures the monitoring and the control of the other SSCs' layers as well as the coordination with the other SSCs of an overall supply chain. Indeed, it analyses the information coming from the Execution System and establishes the necessary metrics. Based on these metrics, it measures the SSC performance and acts on the other layers accordingly. This system must also coordinate decision-making activities with the other SSCs in the supply chain in order to optimize the product flow and to achieve the overall objectives. At the monitoring layer, the flows are managed by different components. These components are the units responsible for the decision-making and control. As presented in Figure 4, the Monitoring System layer is composed of Monitoring Actors (MAs) which can be defined as follows:

- A Monitoring Actor (MA) is the concept that models an intelligent actor. It represents the actors involved in all SSC layers and particularly in the Monitoring System for decision-making activities and the control of the other SSC layers and the overall supply chain. In some cases, some MAs involved in the Execution System could have dual roles or objectives (synchronization / monitoring). 
Figure 4 Monitoring system

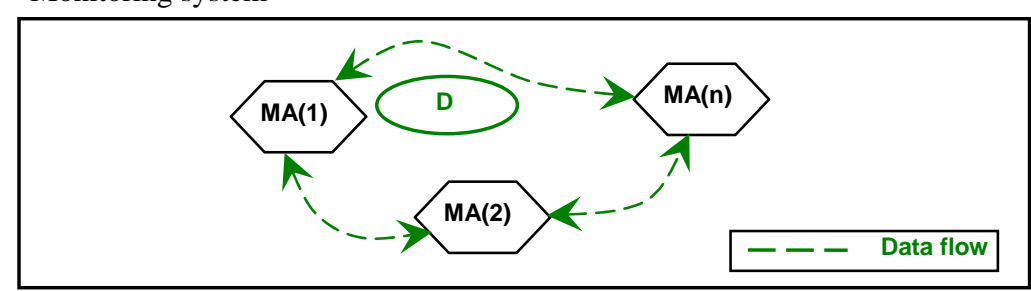

The second layer is the Execution System. It corresponds to the reactive layer of the SSC. Firstly, it synchronizes the operations of the Physical System according to the information flow gathered from this system. Secondly, it is a reactive system; it acts on the Physical System by observing it and its perimeter of influence. In exceptional situations, the Execution System refers to the Monitoring System for coordination and decision-making. The Execution System layer (Figure 5) is composed of two types of actors: the Monitoring Actors (MAs) and the Executive Actors (EAs). These actors apply a reactive control method on the Physical System layer.

- $\quad$ Executive Actors (EAs): the EAs act on the Physical System changes by applying a reactive control. They observe the physical environment and, from the captured perception, they synchronize the operations of the Physical System in order to prevent and/or correct disturbances.

- $\quad$ Monitoring Actors (MAs): a MA in the Execution System is identical to a MA in the Monitoring System. However, in this case, the control activity of a MA is the synchronization of the physical flow.

Figure 5 Execution system

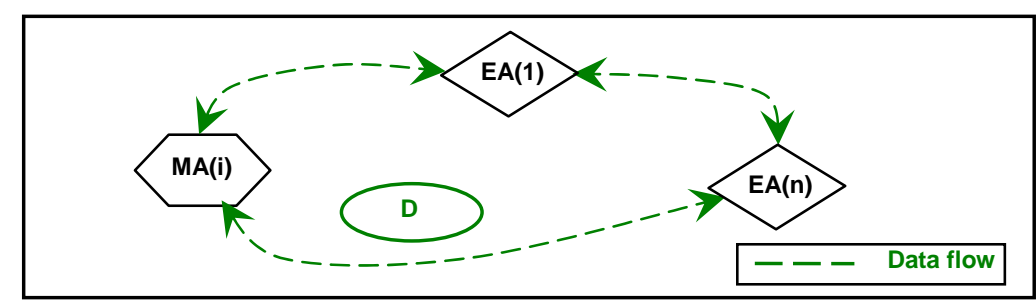

The third layer corresponds to the Physical System. It is the visible part of the environment for a SSC corresponding to its perimeter of influence. It contains the elements that model the physical flow and the resources needed to achieve the SSC's goals or to process an operation. As it is presented in Figure 6, the Physical System is composed of concepts that are passive towards themselves and are controlled by the other layers of a SSC:

- Moving Entity (ME): it models the physical flow (or product) in circulation (motion) into the supply chain.

- $\quad$ Resource (R): this concept models the resources needed for actors to carry out their tasks or activities.

Figure 6 Physical system

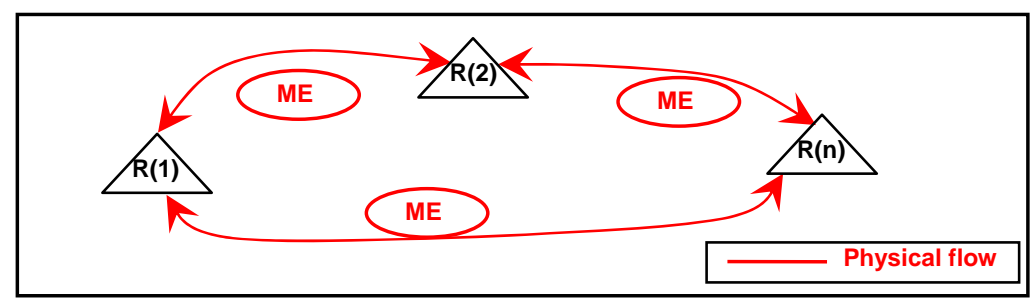




\subsection{Applying "Process Vision"}

During the previous steps of modelling, we built up an intermediary supply chain model by identifying the organization's structures and the environment in which they operate. In the following section, we refine the model by integrating different types of processes. This integration depends on the role and the decision-making of the different systems.

- Monitoring System: into this layer, we identify two kinds of processes that could be described as Monitoring and Control Processes (MCPs) and Strategic Processes (SPs). MCPs are short and medium term management processes: their role is to control the system according to the perceived disturbances, but also to drive and evaluate the SSC's local performance and in the overall supply chain. The SPs, corresponding to the management processes in the long term, appear in the overall supply chain. The decision at that level must be made in coordination and interaction among all the SSCs integrated in the supply chain.

- Execution System: into this layer, we identify the management processes in the short term and they can be described as Operational Control Processes (OCPs). These processes allow the actors to synchronize and control the flow circulation in the physical layer.

- Physical System: into this layer, the Physical Processes (PhPs) correspond to the succession of operations on resources. They define the transformation stages of the product. A PhP can be supported by one or more SMEs.

\subsection{The final conceptual model}

At this step of the methodology, the final conceptual model is built. This model is divided into two main sub-models: the first being the sub-model of the overall supply chain and the second is the sub-model of network organizations involved in the supply chain.

The overall supply chain model focuses on the organizations and processes that coordinate the different activities. This model is presented in Figure 7.

Figure 7 Global supply chain model

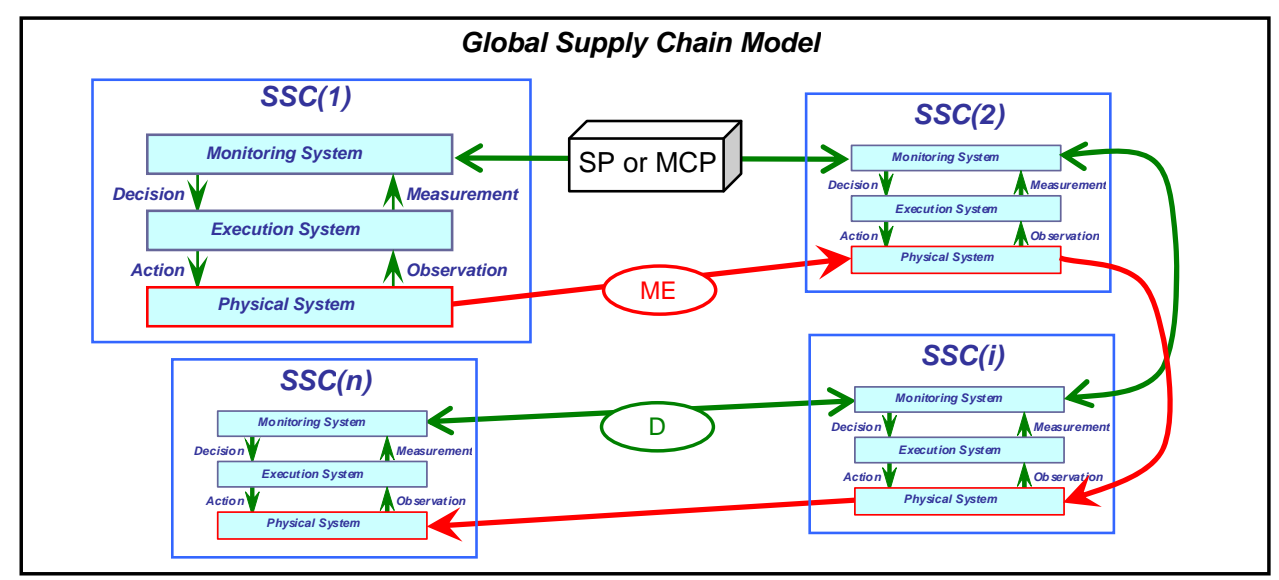

The SSC model corresponds to the structure of the SMEs network as well as the supported processes. This model is presented in Figure 8. 
Figure 8 SSC model

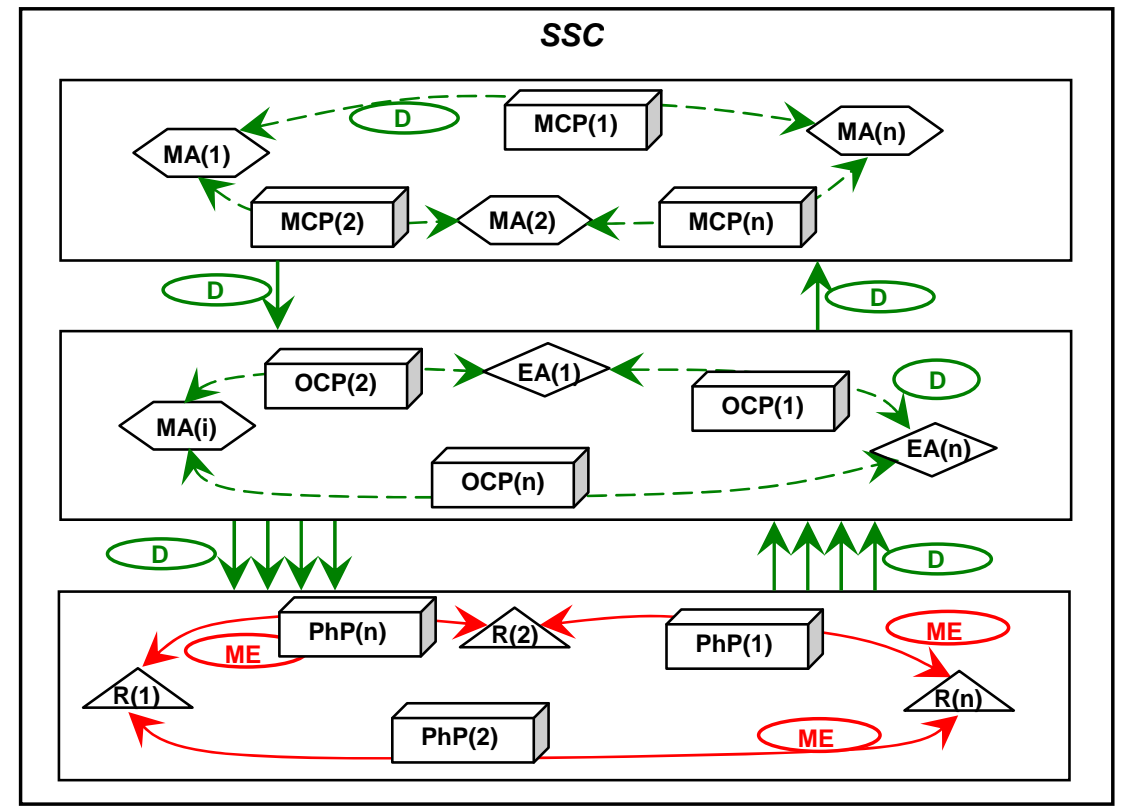

In this section, a conceptual model for a SMEs network in the field of mechatronics has been built. In the following section, we integrate some mechanisms which define some management practices.

\section{Integration of some management mechanisms in the model}

The primary factor of the integration of management practices in a supply chain concerns the use of a communication mechanism to synchronize the different flows (physical flows, information flows and financial flows). In a supply chain, we can find several types of actor relationships depending on the nature of their goals and their degree of involvement. Indeed, Wong et al. (Wong et al., 2004) highlight that in a supply chain, supply chain management is based on three types of relationships: coordination, cooperation and collaboration.

In most cases, these mechanisms of management are used in a similar way to those used in coordination. However, according to the literature, each mechanism has its own characteristics.

Before describing a relationship between two actors in a supply chain, one must consider the criteria relating to: objectives, risks, profits and formal exchanges (Table 1).

Table 1 Communication mechanisms

\begin{tabular}{llll}
\hline & Collaboration & Cooperation & Coordination \\
\cline { 2 - 4 } Objective & Common & Not regrouped & Common \\
Decision-making & Common & Individually & Common + actor decision maker (in such a case) \\
Benefits & Shared & Individually & Shared \\
Resources & Shared & Individually & Individually (but known by others) \\
Formalism & Formal & Few formal & Formal \\
Risks & Shared & Not shared & Shared \\
\hline
\end{tabular}

In the proposed model, we can distinguish two possible relationships. The first one corresponds to the coordination of the activities between several SSCs in the overall supply chain. The second, corresponding to collaboration, is an internal communication mechanism between the actors involved in the same SSC. We have not chosen cooperation as an integration mechanism because it does not meet our 
needs. Indeed, we have based our choice on resources, benefits and risks sharing, and common decisionmaking and objectives.

\subsection{Coordination in the overall supply chain}

SSCs will coordinate their activities in order to achieve the supply chain's overall objectives. At the overall level, each SSC has a limited perimeter of influence. For this reason, a SSC needs to communicate with other SSCs to face the environment disturbance by coordinating their activities and local objectives in order to achieve the overall objective. Coordination will be done through the exchange of the physical flow between Physical Systems in the SSCs. The physical flow exchange is unidirectional. Coordination also occurs between the Monitoring System of SSCs through information flows and performance indicators. In this case, the information exchange is bidirectional. This coordination takes place in the following scenarios:

- Initial configuration of the system: SSCs interact in order to set and coordinate their goals in the overall supply chain.

- Environment disturbance affecting all the goals: if a disturbance occurred in a Physical System of a SSC which requires a modification of its local objective and which affects other SSCs objectives, coordination between SSCs is necessary in order to find an appropriate solution to the problem.

- Reconfiguration of the system: if SSCs recognize that the current system configuration cannot achieve the overall objective, the interaction between SSCs is required to set new objectives according to their experience.

\subsection{Collaboration into the SSC}

The SSC represents a SMEs network collaborating to reach a local objective. The collaborative process takes place according to the following scenarios:

- Physical system synchronization: in the Execution System of the SSC, actors synchronize their information and their actions. This collaboration is frequent in the SSC in commonly encountered situations

- Monitoring SSC: monitoring and control of the SSC will be based on the performance evaluation and the resolution of new disturbances in the physical environment which do not disturb the other SSCs' objectives.

\section{Industrial case}

The case study presented here deals with a SMEs group that acts as mechatronic providers in France and the Benelux countries. The products are manufactured in factories in China and Thailand. Then, they are distributed worldwide by providers such as the one studied.

The production process is invisible to providers. Indeed, in this example, customer needs (forecasts and demands) are gathered annually by decision-making actors in the network. After that, the forecasts are sent to other organizations involved in production planning. The visibility in this supply chain is defined by the geographical location.

In order to be consistent with the conceptual model presented earlier, we consider the example of this supply chain as a node between several sub supply chains (SSCs). Let us call this SMEs network "SSCFrance" and the other networks "SSC-related country". Thus, we get a model of the overall supply chain which is reflected in Figure 9. 
Figure 9 Global supply chain of SSC-France

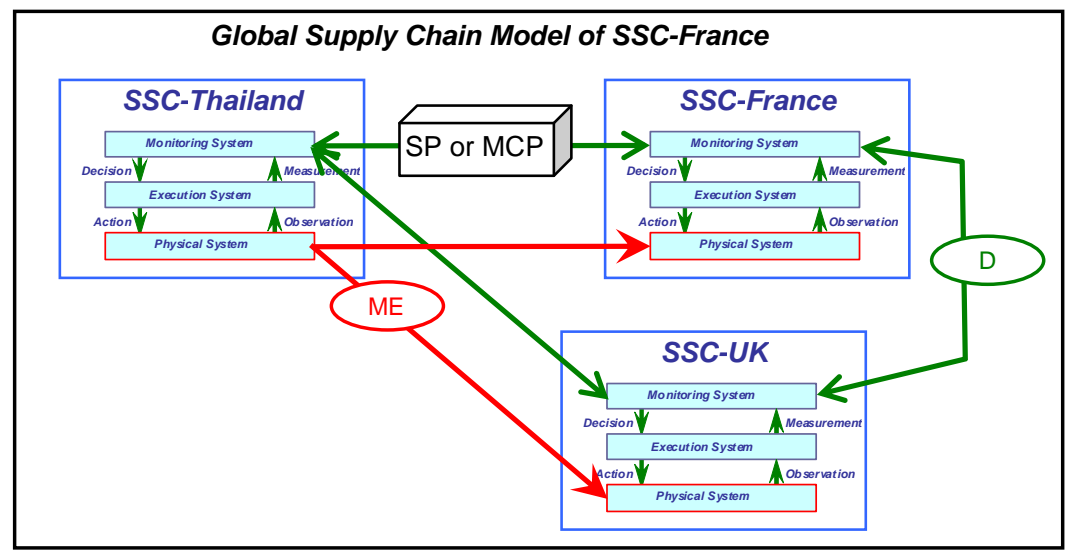

In this model, we have identified the different networks in the supply chain example. Then, we have identified the structure of the SSC-France network and its processes. We have studied the actors of the SSC-France depending on their role and their level of decision-making according to the conceptual model. We have also studied the processes that are supported by SSC-France and have sorted them into the categories of the SSC conceptual model previously identified.

\subsection{SSC-France actors}

In this part, we will categorize the actors in SSC-France according to their role. The different roles as well as the number of actors for each role are listed below:

- Logisticians: they are actors who supervise the flow of cartons from their arrival at Marseille docks until their delivery. They coordinate the activities of the other actors in the SSC, plan the SSC's requirements and monitor the evolution of the physical environment and delivery conditions. They also manage reverse logistics in litigation cases. For this role, we will identify 4 SMEs. Each of them has a specific monitoring role according to the evolution of the processes. We will designate these SMEs L1, L2, L3, and L4.

- $\quad$ Storehouse: these are organizations that support the storage of products and the preparation of orders. In this kind of role, we identify two actors which we will denote S1 and S2.

- Carrier: the SSC deals with 2 transport companies according to the travel mode, destination and type of delivery. We will designate these 2 SMEs by C1 and C2. C1 handles transportation from the warehouse to customers or to another carrier in the case of some Benelux countries. C2 is responsible for transporting cargo from China or Thailand. This transport is done in the case of procurement to meet customer's orders.

\subsection{SSC-France processes}

In this industrial case, we identified many processes which are managed by SSC-France. These processes can be classified into five macro processes (in reference to the SCOR model): plan, distribution, outsourcing, deliver and return management. Each macro process is divided into many processes carried out by one or more actors. Therefore, we will consider four physical processes in our example: PP1, PP2, PP3 and PP4. 


\subsection{The conceptual model of SSC-France}

In this section, we refer to the modelling concepts, an identified entity from the industrial case.

\section{Actors}

L1, L2, and L3: Monitoring Actors (MAs).

S1: Monitoring Actor involved in the Execution System as well (MA).

S2, C1 and C2: Execution Actors (EAs).

\section{Resources}

In our case, we need 3 resources to achieve all the processes.

\section{Processes}

PhP1, PhP2 and PhP3: Physical Processes. In this case, physical processes can be supported by only one resource.

According to this correspondence, the conceptual model of the SSC-France is shown in Figure 10.

Figure 10 Conceptual model of SSC-France

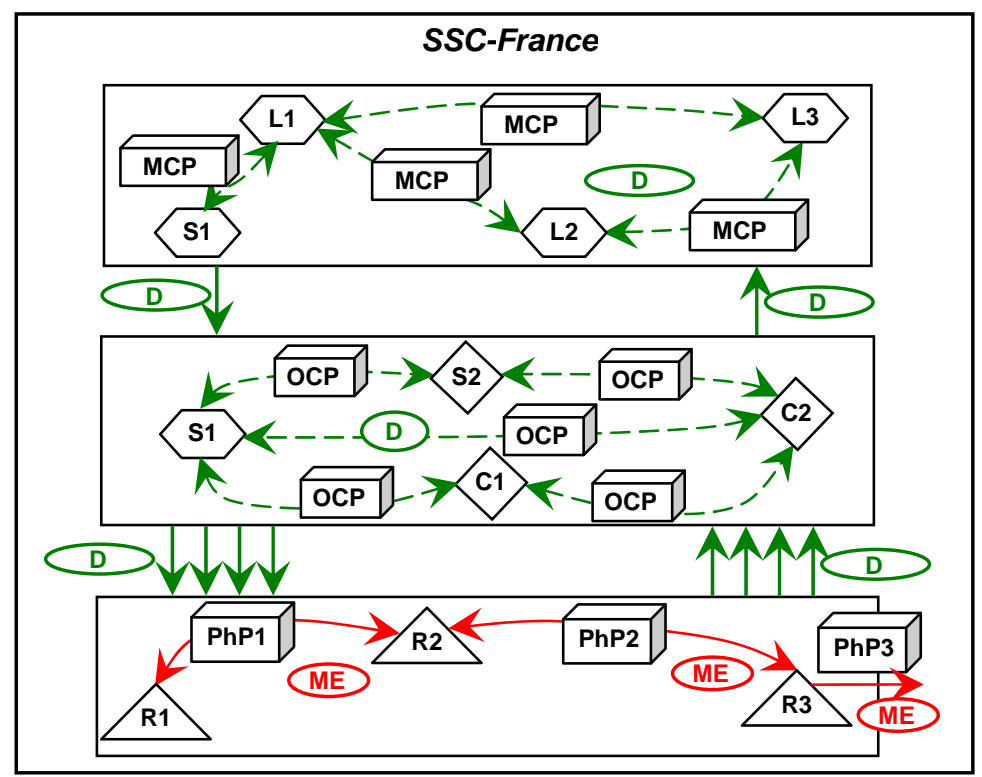

\section{Conclusion and perspectives}

Mechatronic Small and Medium Enterprises integrate complex supply chains whose overall scopes are sometimes not visible. SMEs collaborate to achieve one or more processes in order to respond to the supply chain demands quickly and to survive the environment disturbances.

In this paper, we have analyzed SMEs in a mechatronic environment, developed a modelling methodology and proposed concepts dedicated to supply chain modelling and simulation. This methodology and these concepts were applied to an actual supply chain which is mainly composed of SMEs. We have also identified the communication mechanisms to manage and monitor the SMEs network and the supply chain.

A major perspective for this work that is being carried out at the moment is to study the dynamic behaviour of each concept. The final aim of such research is to implement a simulation platform for supply chains that mostly involve SMEs. 


\section{Acknowledgements}

We thank “Assemblée des Pays de Savoie” (APS) and the laboratory "Systèmes et Matériaux pour la Mechatronic” (SYMME) who fund this work through a research grant.

\section{References}

Assens C., 1994. Du modèle bureaucratique au modèle organique, Research Notebook n²32, DMSP.

Bagchi P.K. and Skjoett-Larsen T., 2005. Supply chain integration: an European survey, International Journal of Logistics Management, vol 16, n 2, pp.275-294.

Bagchi S., Buckley S., Ettl M., Lin G., 1998. Experience using the IBM supply chain simulator. In Proceedings of the winter Simulation Conference.

Christopher, 1992. Logistics and Supply chain managements. Pitman Publishing, London.

Chopra, S. and Meindl, P. 2001. Supply Chain Management: strategy planning and operation. Upper Saddle River, NJ: Prentice-hall.

Cooper M., Lambert D.M. and Pagh J.D., 1997. Supply chain management: more than a new name for logistics. International Journal of Logistics Management, vol 18, $\mathrm{n}^{\circ}$ 2, pp. 1-13.

Drucker P.F, 1998. Management’s new paradigms. Forbes, October, pp. 152-177.

Ingalls R.G, 1998. The value of simulation in modelling supply chain. In Proceedings of the 1998 winter simulation conference, Washington DC, pp. 1371-1375.

Julien P.A., 1997. Les PME bilan et perspectives. 2e edition, Economica, Paris, France.

Kearney A.T, 1994. Management approach to Supply chain Integration. Research Notebook, Chicago.

Labarthe O., Espinasse B., Ferrarini A., Montreuil B., 2007. Toward a methodological framework for agent-based modelling and simulation of supply chains in a mass customization context. Simulation Modelling Practice and Theory, vol. 15, pp.113-136

La Londe B.J and Masters J.M, 1994. Emerging logistics strategies: Blueprint for the next century. International Journal of Physical distribution and Logistics Management, vol. 24, no 7, pp. 35-47.

Lambert D.M. and Cooper M.C., 2000. Issues in supply chain management. Industrial Marketing Management, 29, $\mathrm{n}^{\circ} 1$, pp. 65-83.

Lee Y.H, Cho M.K., Kim S.J. and Kim Y.B, 2002. Supply chain simulation with discrete continuous combined modelling. Computer and Industrial Engineering, 43, 375-392.

Li X. and Wang Q., 2007. Coordination mechanisms of supply chain systems. European Journal of operational Research, vol 179, $\mathrm{n}^{\circ} 1$, pp. 1-16.

Longo F., Mirabelli G., 2008. An advanced supply chain management tool based on modelling and simulation. Computers \& Industrial Engineering, vol 54, pp. 570-588

Mallidi K., Paraskevopoulos A.T. and Pagnelli P., 1999. Process Modelling in small-medium enterprise networks. Computers In Industry, vol 38, n² 2, pp. 149-158.

Min H. and Zhou G., 2002. Supply chain modelling: past, present and future. Computers \& Industrial engineering, vol 43, n 1--2, pp. 231-249.

Rota K., Thierry C., BEL G., 2002. " Supply chain management: a supplier perspective", Production Planning and Control, 13(4), 370-380.

Stevens, G.C. 1989. Integrating the supply chain. International Journal of Physical Distribution and Materials Management, 19, 3-8.

Thierry C. 2003. Gestion des chaînes logistiques : Modèle et mise en œuvre pour l’aide à la décision à moyen terme. Accreditation to supervise research. University of Toulouse II.

Villarreal Lizarraga, C.L., Dupont L., Gourg D., Pingaud H., 2005. Contributing to management of shared projects in SMEs manufacturing clusters. 18th International Conference on Production Research (ICPR-18), Salerno, Italie.

Wong C.Y., Johansen J. and Hvolby H.H., 2004. Supply chain coordination Problems: Litterature Review from organization, economic and operations perspectives. Working Paper no. 08-04, Center for Industrial Production, Aalborg University, pp. 1-23 